\title{
Transcranial direct current stimulation improves myofascial pain syndrome and chronic fatigue
}

\author{
R.G. Misse, A.M. dos Santos, J.M. de Souza, S.K. Shinjo \\ Division of Rheumatology, Faculdade de Medicina FMUSP, Universidade de Sao Paulo, \\ Sao Paulo, SP, Brazil
}

$\mathrm{D}$ ear Editor,

Myofascial pain syndrome (MPS) is usually accompanied by fatigue, mood alterations, and impaired quality of life (1). MPS treatments include pharmacological regimens (e.g., analgesics, muscle relaxants) and non-pharmacological schemes (e.g., exercise training, physiotherapy, acupuncture). Most of these strategies can also be applied to the treatment of fatigue. In addition, transcranial direct current stimulation (tDCS) has been used with good outcomes in individuals with MPS $(1,2)$ or fatigue $(3,4)$. The tDCS is safe with no serious adverse effects, and can reserve the central pain pathway by modulating cortical plasticity (2-4). To the best of our knowledge, no studies have assessed the effects of tDCS in patients with both refractory MPS and chronic fatigue.

In this context, we assessed a 33-year-old non-sedentary female patient with refractory MPS and fatigue, which had lasted for two years. She had received several therapies, such as gabapentin, amitriptyline, acupuncture, and Pilates exercises, which consists of low-impact flexibility and muscle strength exercises, and improve the overall body coordination. At admission to our service, she had a trigger point in her right quadratus lumborum, which radiated pain throughout the gluteus medius. Moreover, she had a trigger point in her left sternocleidomastoid, trapezius, levator scapulae, and posterior cervical muscles, which caused radiating pain throughout to her entire left arm and shoulder. These trigger points were defined as sensitive spots and were located in a taut band of skeletal muscles. They caused local pain and referred muscle pain.

After signing the informed consent, the patient underwent five consecutive tDCS sessions with $2 \mathrm{~mA}$ for 20 minutes. The positively-charged electrode was positioned at C3 (contralateral to the dominant limb), while the negatively-charged electrode was placed in the supraorbital region FP2. During the tDCS sessions, the patient performed also some physical exercise, which included a 5-minute treadmill warm-up followed by lower intensity (5) strength exercises: bench press, bench-squat, cable seated row, and leg press at $45^{\circ}$ (three sets of 10-12 repetitions with breaks between 45 and 60 seconds).

At baseline (PRE) and after five days (POST), the patient completed the following questionnaires: Pain Quality Assessment Scale (PQAS) (6), McGill pain inventory (7), Fatigue Severity Scale (FSS) (8), and Short Form-36 (9). The data were expressed as percentages (\%) of change: (the difference between post and pre-values)/ pre-value.

Compared to the PRE phase, myofascial pain decreased in the POST phase, as shown through the PQAS and McGill pain inventory questionnaires (Figure 1A and $1 \mathrm{~B})$. Moreover, notable improvements were observed in all the domains of quality of life (Figure 1C). In addition, there was a 


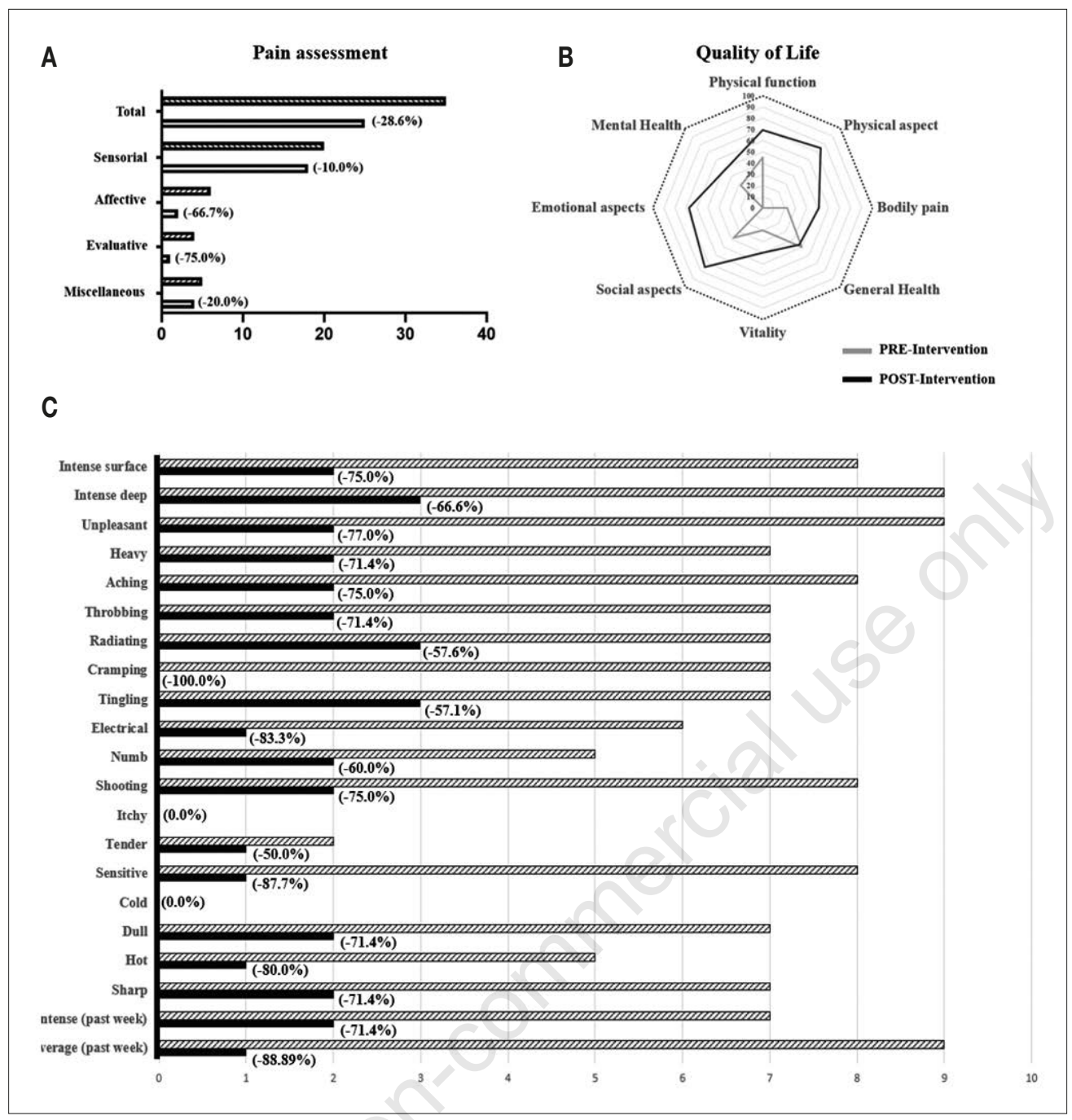

Figure 1 - Data related to McGill pain assessment (A), health quality of life $(B)$, and $(C)$ general pain quality assessment. The data were expressed as percentages (\%) of change: (the difference between post and pre-values)/pre-value.

mild reduction in the FSS parameters: 6.1 vs. $5.7(-6.6 \%)$. There was no adverse effect related to tDCS and exercise training, and the patient participated in all phases of the protocol.

According to the literature, our patient had a good outcome with tDCS associated with exercise training: pain and fatigue decreased and quality of life also improved. These results can be related to the impact on the maladaptive efferent feedback mechanism present in pain, which leads to consequent reduction of fatigue (4).

These results are promising, but further studies with a larger sample size are necessary to confirm our findings.

\section{Funding}

This work was funded by: Fundação de Amparo à Pesquisa do Estado de São Paulo (FAPESP) \#2019/12155-5 to RGM, \#2018/08735-3to AMS, and \#2019/117766 to SKS; Conselho Nacional de Desenvolvimento Científico e Tecnológico (CNPq) 303379/2018-9 to SKS; Faculdade de Medicina da USP to SKS.

\section{Conflict of interest}

All authors declare no conflict of interest. 


\section{REFERENCES}

1. Saxena A, Chansoria M, Tomar G, Kumar A. Myofascial pain syndrome: an overview. J Pain Palliative Care Pharmacother. 2015; 29: 16-21.

2. Fagerlund AJ, Hansen OA, Aslaksen, PM. Transcranial direct current stimulation as a treatment for patients with fibromyalgia: a randomized controlled trial. Pain. 2015; 156; 62-71.

3. Choi YH, Jung SJ, Lee CH, Lee SU. Additional effects of transcranial direct-current stimulation and trigger-point injection for treatment of myofascial pain syndrome: a pilot study with randomized, single-blinded trial. J Altern Complem Med. 2014; 20: 698-704.

4. Lefaucheur JP, Chalah MA, Mhalla A, Palm U, Ayache SS, Mylius V. The treatment of fatigue by non-invasive brain stimulation. Neurophysiologie Clinique. 2017; 47: 173-184.
5. Borg G. Psychophysical scaling with applications in physical work and the perception of exertion. Scand J Work Environ Health. 1990; 16: $55-58$.

6. Carvalho AB, Garcia JBS, Silva TKM, Ribeiro JVF. Tradução e adaptação transcultural da Pain Quality Assessment Scale (PQAS) para a versão brasileira. Braz J Anesthesiol. 2016; 66: 94-104.

7. Melzack R. The McGill Pain Questionnaire: major properties and scoring methods. Pain. 1975; 1: 277-99.

8. Krupp LB, LaRocca NG, Muir-Nash J, Steinberg AD. The fatigue severity scale. Application to patients with multiple sclerosis and systemic lupus erythematosus. Arch Neurol. 1989; 46: 1121-3.

9. Da Mota Falcão D, Ciconelli RM, Ferraz MB. Translation and cultural adaptation of quality of life questionnaires: an evaluation of methodology. J Rheumatol. 2003; 30: 379-85. 